\title{
Pattern of the Antimalarials Prescription during Pregnancy in Bangui, Central African Republic
}

\author{
Alexandre Manirakiza, ${ }^{1,2}$ Georges Soula, ${ }^{2}$ Remi Laganier, ${ }^{1}$ Elise Klement, ${ }^{3}$ Djibrine Djallé, ${ }^{1}$ \\ Moyen Methode, ${ }^{4}$ Nestor Madji, ${ }^{4}$ Luc Salva Heredeibona, ${ }^{5}$ Alain Le Faou, ${ }^{6}$ and Jean Delmont ${ }^{2}$ \\ ${ }^{1}$ Institut Pasteur of Bangui, Epidemiology Service, P.O. Box 923, Pasteur Avenue, Bangui, Central African Republic \\ ${ }^{2}$ Centre de Formation et de Recherche en Médecine et Santé Tropicales, Faculté de Médecine Nord, Boulevard Dramard, \\ 13015 Marseille, France \\ ${ }_{3}^{3}$ Association Altersanté, Centre Médical de Bligny, 91640 Briis-sous-Forges, France \\ ${ }^{4}$ Ministère de la Santé, de la Population et de Lutte Contre le VIH-SIDA, National Malaria Control Service, \\ P.O. Box 143, Central African Republic \\ ${ }^{5}$ Ministère de la Santé, de la Population et de Lutte Contre le VIH-SIDA, Health Centre of Castors, Bangui, \\ P.O. Box 143, Central African Republic \\ ${ }^{6}$ Université de Nancy, CHU de Nancy, Hôpital de Brabois-adultes, 54511 Vandoeuvre-lès-Nancy Cedex, France
}

Correspondence should be addressed to Alexandre Manirakiza, amanirak@yahoo.fr

Received 6 February 2011; Accepted 8 June 2011

Academic Editor: Valery Combes

Copyright (C) 2011 Alexandre Manirakiza et al. This is an open access article distributed under the Creative Commons Attribution License, which permits unrestricted use, distribution, and reproduction in any medium, provided the original work is properly cited.

\begin{abstract}
Introduction. The aim of this study was to identify the antimalarials prescribed during the pregnancy and to document their timing. Method. From June to September 2009, a survey was conducted on 565 women who gave birth in the Castors maternity in Bangui. The antenatal clinics cards were checked in order to record the types of antimalarials prescribed during pregnancy according to gestational age. Results. A proportion of $28.8 \%$ ANC cards contained at least one antimalarial prescription. The commonest categories of antimalarials prescribed were: quinine (56.7\%), artemisinin-based combinations (26.8\%) and artemisinin monotherapy $(14.4 \%)$. Among the prescriptions that occurred in the first trimester of pregnancy, artemisinin-based combinations and artemisinin monotherapies represented the proportions of (10.9\%) and (13.3\%). respectively. Conclusion. This study showed a relatively high rate $(>80 \%)$ of the recommended antimalarials prescription regarding categories of indicated antimalarials from national guidelines. But, there is a concern about the prescription of the artemisinin derivatives in the first trimester of pregnancy, and the prescription of artemisinin monotherapy. Thus, the reinforcement of awareness activities of health care providers on the national malaria treatment during pregnancy is suggested.
\end{abstract}

\section{Introduction}

Malaria in pregnancy is a serious public health problem in endemic regions. It is estimated that 85 millions pregnancies occurred in Plasmodium falciparum malaria-endemic areas [1]. This pathology is among the main causes of the low birth weight (LBW) $[2,3]$, resulting from $3 \%$ to $8 \%$ neonatal and infant deaths [3]. This led the World Health Organization (WHO) to recommend a package of affordable interventions including the use of the insecticide-treated nets (ITNs), the intermittent presumptive treatment (IPT) with sulfadoxinepyrimethamine (SP), and the effective case management of clinical malaria with recommended antimalarials. The IPT with SP (IPT-SP) is given free of charge under direct observed therapy (DOT) during the antenatal clinics (ANCs) at regular predefined intervals of time [4], with the aim to achieve the clearance of the existing Plasmodium falciparum asymptomatic infection (therapeutic effect) and the suppression of new maternal infections (prophylactic effect) over a period of time [5]. At least two doses of IPT-SP are recommended during the second and third trimesters of pregnancy [4]. The occurring of clinical malaria should be treated with quinine or artemisinin-based combinations. Quinine is considered to be a safe drug along all the period 
of the pregnancy, but safety data of artemisinin derivatives during the first trimester of pregnancy are limited [4], thus it should not be prescribed in this period.

The integration approaches to these programmatic strategies consist on the training and the supervision. Ongoing assessments on the compliance of health care providers with national policy on malaria treatment are needed. Actually, no published data on prescribing practices in accordance to these new strategies are available in Central African Republic (CAR) and elsewhere.

Since 2006, the CAR malaria control programme is implementing the WHO new guidelines on malaria management $[4,6]$. The training seminars of the health care providers (nurses, midwives and midwives assistants, medical officers) from public and private health centers were achieved. The supervision and the field training visit are scheduled each year at each health centre.

The aim of this study was to design a preliminary assessment on the compliance of a trained staff in the malaria control new strategies at the main district maternity in Bangui. Thus, we identified the antimalarials prescribed for possible clinical malaria episodes during the pregnancy and the timing profile of those prescriptions according the gestational age.

\section{Methods}

2.1. Study Design and Population. Our study was conducted from June to September 2009 at one of the two main maternities of Bangui city, the maternity of the Castors government health centre. This health centre is located in the most populated area of the city and is purposely selected during this study as a representative pilot center for the practice of the trained health care providers in Bangui, regarding antimalarials prescription to pregnant women. The study involved all women who gave birth at the Castors Health Centre maternity, and who have been clinically followed up in the antenatal care services at this same health centre. In April 2006, the ANC staff ( 2 midwives and 6 midwives assistants) has baseline training in the malaria control new strategies and is regularly supervised once per year accordingly. This was the key indication for us to assess how the implementation of the new prescribing recommendations is progressing. The average proportion of pregnant women in Bangui who deliver in this maternity each year is estimated at 35\% (2500 deliveries) [7], and more than $95 \%$ of those women have their ANC visits in this health centre (data checked from the centre registers).

After delivery, all the women from whom we got a written informed consent were immediately approached to record the prescribed antimalarials from their antenatal clinics (ANC) cards. Women who have not done all their ANC visits at the Castors Health Centre were excluded from this study. All the SP records were the IPT-SP, which is the only antimalarial treatment given free of charge to the pregnant women (direct observed therapy or DOT).

2.2. Ethical Approval. This study was undertaken during a preliminary assessment of our project aiming at estimating the burden of HIV infection on the malaria prevention effectiveness during pregnancy in CAR. This project was reviewed and approved by the Scientific Committee Board of The University of Bangui in charge of the validation of scientific protocol studies in CAR. This study received the agreement of the University of Marseille, France.

2.3. Data Analysis. Data were entered in Epilnfo software version 3.5.1 (CDC, Atlanta, USA). An investigator checked the EpiInfo data base and corrected errors identified after referring to questionnaires. Data consistency was also verified using Excel software (Microsoft Corporation), and statistical analysis was achieved in Stata 8.0 (StataCorp LP, USA). Findings are presented as frequencies and proportions. Proportions were compared with the $\chi^{2}$-test (or Fisher test), and a $P$ value at less than 0.05 was considered statistically significant.

\section{Results}

A total of 565 women were enrolled for this study. Their mean $( \pm$ SD) age was $24.7( \pm 6.0)$ years (range: 15 to 45 years). A proportion of $21.8 \%(n=123)$ has been given at least one dose of IPT-SP. Only a proportion of $8.5 \%$ of them was given more than one dose of IPT-SP, as is recommended by the malaria control programme.

3.1. Prescribed Antimalarials. Overall, 163 pregnant women $(28.8 \%)$ were prescribed at least once an antimalarial for a possible clinical malaria episode. The number of antimalarials prescription ranged from 1 to 3 times per ANC cards. Eight ANC cards contained 3 prescriptions (4.9\%), and 29 ANC cards contained 2 prescriptions (17.8\%). Overall, four different groups of antimalarials were prescribed to the 163 women during the pregnancy, and the total number of those prescriptions was 208 (the mean number of antimalarials prescription per women was 1.28): quinine (56.7\%), artemisinin-based combinations $(26.8 \%)$, artemisinin monotherapies (14.4\%), and other antimalarials monotherapies (chloroquine, halofantrine, and amodiaquine). The malaria laboratory microscopy diagnosis was available in the ANC cards for only $18.9 \%$ antimalarials prescriptions (39/208). All those results were positive for a Plasmodium falciparum, and the quinine was the only antimalarial prescribed accordingly.

\subsection{Profile of Antimalarials Prescribed according to Gestational} Age. Antimalarials prescription began in the second month of gestation, reaching a peak at the sixth month of gestation. The global proportion of those prescriptions significantly increased from the first trimester $(16.8 \%)$ to the second trimester $(56.3 \%)\left(P\right.$ value $\left.<10^{-6}\right)$. There were a total of 85 artemisinin components prescriptions during the current pregnancies ( 30 artemisinin monotherapies and 55 artemisinin-based combinations). Among those prescriptions, four (13.3\%) prescriptions of artemisinin monotherapies and six (10.9\%) artemisinin-based combinations prescriptions occurred in the first trimester of pregnancy. Otherwise, we recorded a total number of 118 quinine prescriptions. 


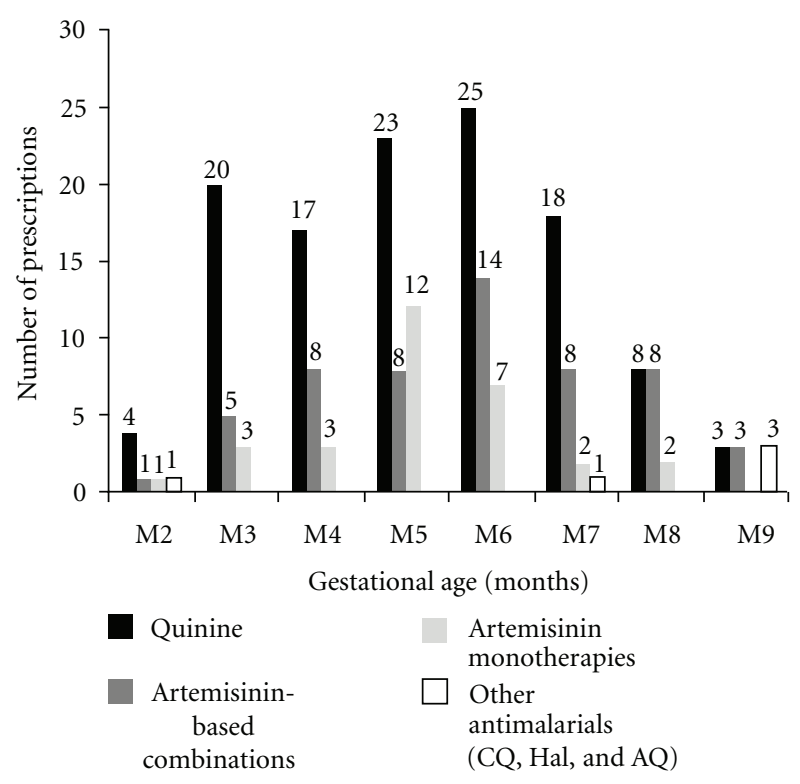

FIGURE 1: Distribution of antimalarials prescribed during the current pregnancy in the maternity of the Castors, Bangui, Central African Republic: June to September 2009. Legend: CQ = Chloroquine, $\mathrm{Hal}=$ Halofantrine, and $\mathrm{AQ}=$ Amodiaquine

The number of quinine prescription in the first trimester is $24 / 35(68.6 \%)$ and in the second trimester $65 / 117(55.5 \%)$, while artemisinin-based combinations are $6 / 35$ (17.1\%) for the first trimester and 40/117 for the second, and artemisinin monotherapy is $4 / 35(11.4 \%)$ for the first trimester and $22 / 117(18.8 \%)$ for the second.

Within each category of antimalarials, the distribution trend increased from the first trimester to the second trimester: $24 / 118(20.3 \%)$ to $65 / 118(55.1 \%)$ for quinine, $6 / 55(10.9 \%)$ to $30 / 55(54.5 \%)$ for artemisinin-based combinations, and $4 / 30(13.3 \%)$ to $22 / 30(73.3 \%)$ for artemisinin monotherapies.

Details on the trend of those antimalarials prescription according to pregnancy age are showed on Figure 1 (see Supplementary Material available online at doi: 10.4061/ 2011/414510).

\section{Discussion}

This study showed that nearly three years following the change in national malaria control strategies during pregnancy, more than $80 \%$ of prescriptions represented the recommended antimalarials (the quinine and artemisininbased combinations). The majority of pregnant women were prescribed the quinine. The availability and affordability of this antimalarial in the health centre pharmacies (a cure costs less than 2 US\$) could have motivated this preference. The quinine is also preferred by the prescribers during malaria management practices in Bangui, and the predominant argument is the prompt evidence of its activity on malaria symptoms [8].

Antenatal exposures to antimalarial drugs during the pregnancy are a perpetual concern because there is limited information on their safety. Indeed, pregnant women are systematically excluded from clinical trials, hence the lack of evidence bases on the toxicity of antimalarial drugs in this population. Nevertheless, the obligation to treat (to save lives) led to the use of antimalarials drugs during pregnancy, as the experts agreed on the safety of the quinine along all the period of the pregnancy and the artemisininbased combinations during the two last trimesters of the pregnancy [6]. Thus, the prescription of the artemisinin derivatives which occurred in the first trimester of pregnancy is relevant because it is not advisable $[4,9]$ and may cause embryotoxicity [10] or enhance embryolethality risks [11].

This study showed also that health care providers prescribed the artemisinin monotherapy. Thus, this regimen is no longer recommended by WHO due to its short plasmatic half-life, which could enhance the spreading of parasite resistance and compromise the efficacy of artemisininbased combinations [12]. It is also surprising that there were 3 prescriptions of the chloroquine which has been withdrawn from the CAR market because of its lack of efficacy on malaria [8]. This could be interpreted as a serious prescription mistake.

This study has some limitations. The first limitation was the method used to record the prescriptions of antimalarial drugs from solely ANC cards. Indeed, other antimalarials taken by the women during their current pregnancies could not have been reported in the ANC cards. However, recording antimalarials use through interview could lead to error because some pregnant women may not have accurately recalled the name of the antimalarial prescribed and/or taken, thus leading to biased findings on the health staffs compliance with the national recommended guidelines. The second limitation is that laboratory malaria analysis results were poorly reported at the ANC visits cards. Thus, we hypothesize that there has been a wide proportion of presumptive treatment [13]. Indeed, the clinical symptoms have not always the positive predictive value of malaria $[14,15]$. Another limitation is that the findings from this study could be different in other health maternities centres due to changes of staff (new health providers), or specificities related to prescribing habits.

\section{Conclusion}

The findings of this study from a district maternity in Bangui provide the indicator of the antimalarials groups prescribed to pregnant women, during the period of the new malaria treatment guidelines implementation in CAR. The observation from this study is that there is a satisfying adherence by trained health care providers to the indicated categories of antimalarials during pregnancy from the ANC staff of the Castors Health Center of Bangui. Nevertheless, there are some errors of antimalarials prescription related to the period of gestation and to not recommended antimalarials prescriptions. The activities focusing on the supervision of health care providers should be reinforced to assure adequate malaria treatment during pregnancy according to national guidelines. In particular, our findings helped the National Malaria programme to enhance supervision activities to the 
staff of Castors Maternity Centre in Bangui in order to limit the prescription errors in the future. Further studies assessing the indicators of the national antimalarial policy implementation to the pregnant population are needed in other areas of the country.

\section{Authors' Contributions}

A. Manirakiza, J. Delmont, and A. Le Faou conceived the study with substantial contributions of R. Laganier, M. Methode, and N. Madji. Field data collection was achieved by A. Manirakiza and L. S. Heredeïbona. Analysis, interpretation of data, and this paper draft were achieved by A. Manirakiza, J. Delmont, D. Djallé, G. Soula, E. Klement, and A. Le Faou.

\section{Conflict of Interests}

The authors declared that there is no conflict of interests.

\section{Acknowledgments}

The authors are grateful to all the women who participated in this study. They also thank the staff of the maternity clinics of Castors in Bangui, where the study was conducted, who kindly collaborated in data collection: Mss. Joséphine Basat, Julienne Grekozo, Léa Guedoukpou, Christine Herebaï, Jeanne Marie Morouba, and Lydie Oulebona. They are very thankful to Joachim Guelembi, $\mathrm{PhD}$, for his precious contributions.

\section{References}

[1] S. Dellicour, A. J. Tatem, C. A. Guerra, R. W. Snow, and F. O. Ter Kuile, "Quantifying the number of pregnancies at risk of malaria in 2007: a demographic study," PLoS Medicine, vol. 7, no. 1, Article ID e1000221, 2010.

[2] C. Menendez, J. Todd, P. L. Alonso, S. Lulat, N. Francis, and B. M. Greenwood, "Malaria chemoprophylaxis, infection of the placenta and birth weight in Gambian primigravidae," Journal of Tropical Medicine and Hygiene, vol. 97, no. 4, pp. 244-248, 1994.

[3] R. W. Steketee, J. J. Wirima, A. W. Hightower, L. Slutsker, D. L. Heymann, and J. G. Breman, "The effect of malaria and malaria prevention in pregnancy on offspring birthweight, prematurity, and intrauterine growth retardation in Rural Malawi," American Journal of Tropical Medicine and Hygiene, vol. 55, no. 1, pp. 33-41, 1996.

[4] WHO, "A strategic framework for Malaria prevention and control during pregnancy in the African Region," 2010, http://whqlibdoc.who.int/afro/2004/AFR_MAL_04.01.pdf.

[5] N. J. White, "Intermittent presumptive treatment for malaria," PLoS Medicine, vol. 2, article e3, 2005.

[6] WHO, "Guidelines for the treatment of malaria," 2006, http:// whqlibdoc.who.int/publications/2006/9241546948_eng_full .pdf.

[7] CAR, "Ministère de la santé publique de la population et de lutte contre le VIH-SIDA," Rapport de santé, 2008.

[8] A. Manirakiza, S. P. Njuimo, A. Le Faou, D. Malvy, and P. Millet, "Availability of antimalarial drugs and evaluation of the attitude and practices for the treatment of uncomplicated malaria in bangui, Central African Republic," Journal of Tropical Medicine, vol. 2010, Article ID 510834, 2010.

[9] WHO, "Assessment of the safety of artemisinin compounds in pregnancy 2007," 2009, http://apps.who.int/tdr/svc/publications/tdr-research-publications/artemisinin-compoundspregnancy].

[10] R. L. Clark, S. A. Lerman, E. M. Cox, W. E. Gristwood, and T. E. White, "Developmental toxicity of artesunate in the rat: Comparison to other artemisinins, comparison of embryotoxicity and kinetics by oral and intravenous routes, and relationship to maternal reticulocyte count," Birth Defects Research Part B, vol. 83, no. 4, pp. 397-406, 2008.

[11] T. E. White, P. B. Bushdid, S. Ritter, S. B. Laffan, and R. L. Clark, "Artesunate-induced depletion of embryonic erythroblasts precedes embryolethality and teratogenicity in vivo," Birth Defects Research Part B, vol. 77, no. 5, pp. 413-429, 2006.

[12] P. Olliaro, "Drug resistance hampers our capacity to roll back malaria," Clinical Infectious Diseases, vol. 41, no. 4, supplement 4, pp. S247-S257, 2005.

[13] T. E. Taha, "Comparison of reported and confirmed malaria during pregnacy: findings from hospital and community studies in Sudan," East African Medical Journal, vol. 73, no. 9, pp. 571-574, 1996.

[14] A. Bardají, B. Sigauque, L. Bruni et al., "Clinical malaria in African pregnant women," Malaria Journal, vol. 7, article no. 27, 2008.

[15] C. Rogier, T. Fusai, B. Pradines, and J. F. Trape, "Evaluating malaria attributable morbidity in endemic areas," Revue d'Epidemiologie et de Sante Publique, vol. 53, no. 3, pp. 299309, 2005. 


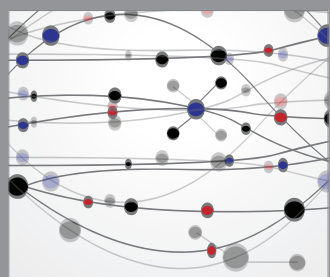

The Scientific World Journal
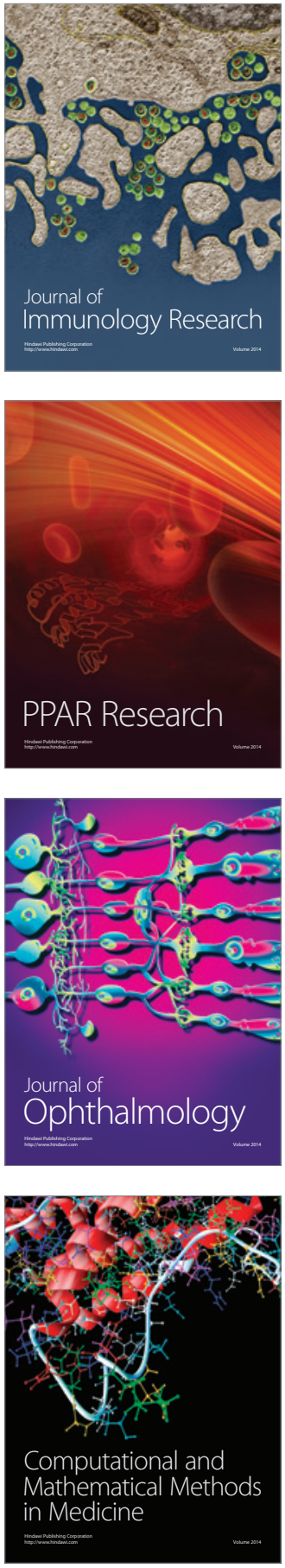

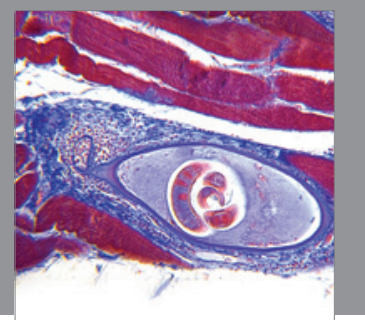

Gastroenterology

Research and Practice
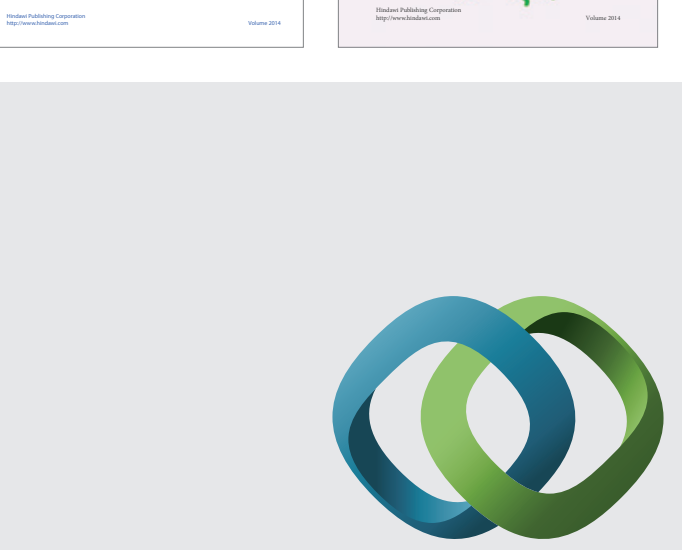

\section{Hindawi}

Submit your manuscripts at

http://www.hindawi.com
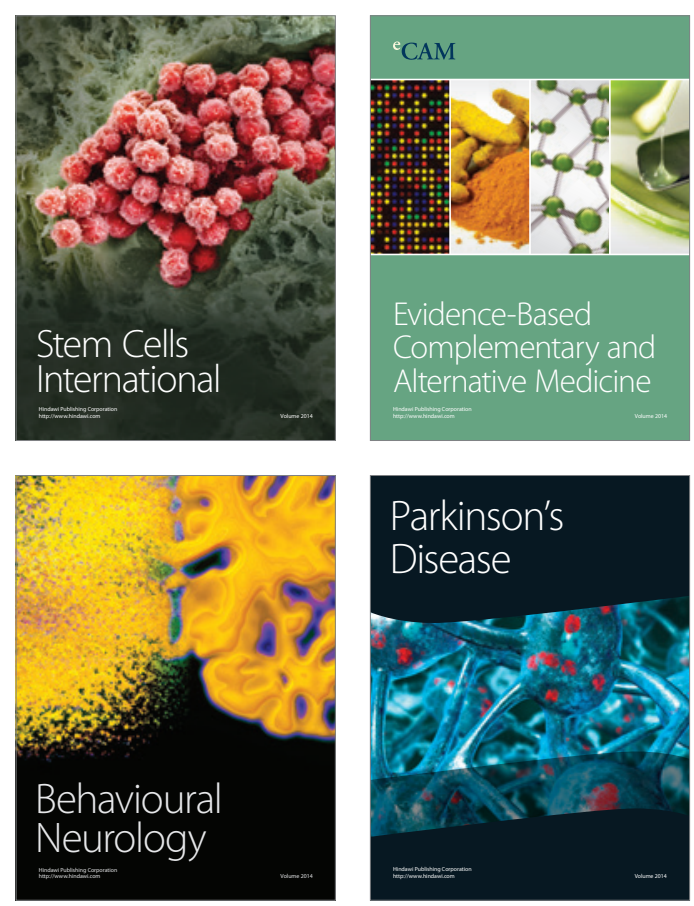

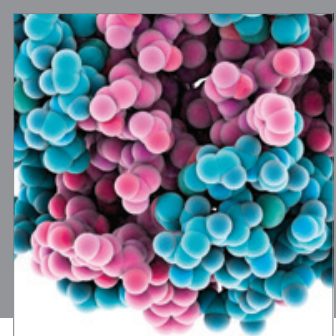

Journal of
Diabetes Research

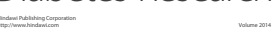

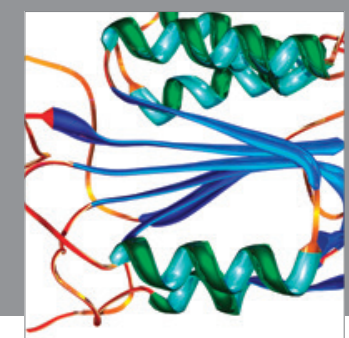

Disease Markers
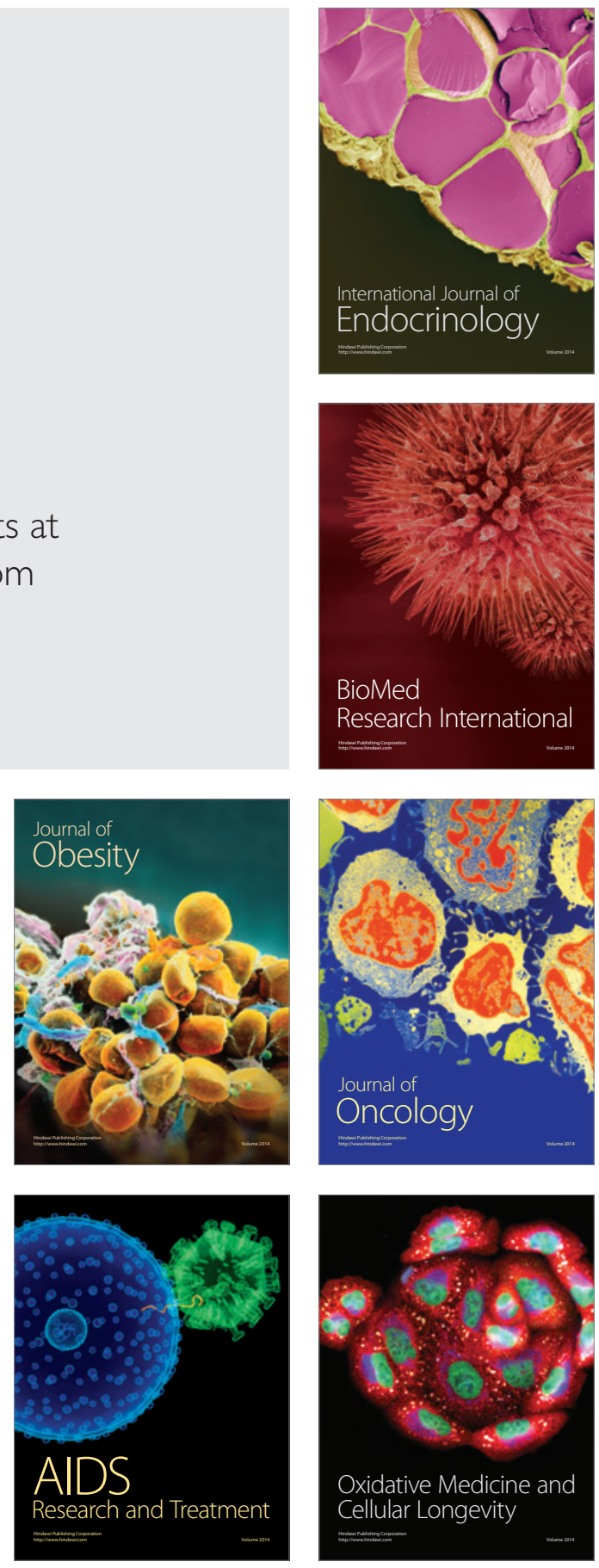